\title{
Antibody-fused interferons as an effective approach to enhance target specificity and antiviral efficacy of type I interferons
}

Lucy S Cheng ${ }^{1}$, Seungmin Hwang ${ }^{2}$, Tzu-Hsuan Huang ${ }^{1}$, Ting-Chang Sheu ${ }^{1}$, Ren Sun $^{2}$, Sherie L Morrison ${ }^{1}$, Genhong Cheng ${ }^{1}$, Arash Shahangian ${ }^{1}$

${ }^{1}$ Department of Microbiology, Immunology and Molecular Genetics, University of California, Los Angeles, 10833 Le Conte Avenue, 8-240 Factor, Los Angeles, CA 90095, USA; ${ }^{2}$ Department of Molecular and Medical Pharmacology, UCLA David Geffen School of Medicine, Los Angeles, CA 90095, USA

Cell Research 18:1230-1232. doi: 10.1038/cr.2008.304; published online 11 November 2008

\section{Dear Editor,}

Interferons (IFNs) were first described for their ability to protect cells from viral infections [1]. Since these initial reports, three major types of IFNs have been described. Type I IFNs, including IFN $\beta$ and multiple IFN $\alpha$ subtypes, induce antiviral gene programs through the IFN $\alpha / \beta$ receptor, IFNAR; many of these genes are directly or indirectly involved in curbing viral replication and spread [2]. Type I IFNs have been used to treat both chronic viral infections, such as hepatitis B and $\mathrm{C}$, and a variety of neoplastic conditions, such as melanoma, hairy cell leukemia, and non-Hodgkin's lymphoma [3-7]. Recently, PEGylated IFNs, which show decreased clearance as compared to recombinant IFNs, have emerged as the standard of care. Although PEGylation has increased the therapeutic efficacy of IFN therapy, a wide variety of unpleasant and serious side-effects exist. In this study, we set out to determine whether antibody-IFN fusions could be used as an alternative and allow for specific targeting of IFNs, which we believe would be beneficial in reducing undesired side-effects.

We have previously generated chimeric molecules by fusing murine IFN $\alpha$ to the carboxyl-terminus of human IgG3 (IgG3-IFN $\alpha$ ) [8]. As IFN $\alpha$ is a potent antiviral cytokine, we examined the ability of IgG3-IFN $\alpha$ to activate the antiviral response and inhibit viral replication.

Correspondence: Genhong Cheng

Tel: +1-310-825-8896; Fax: +1-310-206-5553

E-mail: gcheng@mednet.ucla.edu
Phosphorylated Stat1 was detected in 38C13 cells stimulated with $1 \mu \mathrm{g}$ of IgG3-IFNa for $60 \mathrm{~min}$ (Figure 1A). A recombinant MHV-68 virus (MHV-68-Luc), in which the firefly luciferase gene under the control of the viral M3 promoter is integrated into the viral genome, serves as a convenient readout for viral replication in cultured cells and mice. To determine the ability of IgG3-IFN $\alpha$ to inhibit MHV-68 replication, 38C13 cells were infected with MHV-68-Luc and then treated with either IgG or IgG3-IFN $\alpha$. IgG3-IFN $\alpha$ inhibited viral replication as measured by luciferase activity 2 days after infection (data not shown). To compare the antiviral efficiency of IgG3-IFN $\alpha$ with that of IFN $\alpha, 38 \mathrm{C} 13$ cells were infected with MHV-68-Luc virus and then treated with IFNa or IgG3-IFN $\alpha$ at the indicated concentrations. Luciferase activities were measured 2 days after infection. All experiments were performed in triplicate and repeated at least three times. As shown in Figure 1B, IgG3-IFNa was more effective in inhibiting viral protein expression across a wide range of concentrations.

In addition to increasing the potency, a potential advantageous feature of antibody-conjugated type I IFN is the possibility of using the antibody specificity to target type I IFN to specific cells. Therefore, we used antiHER2-IgG3-IFN $\alpha$, in which IFN $\alpha$ is fused to a HER2/ $n e u$-specific antibody, and $38 \mathrm{C} 13$ cells stably expressing the HER2/neu receptor (38C13-HER2). 38C13-HER2 cells infected with MHV-68-Luc were treated with IgG3IFN $\alpha$ or anti-HER2-IgG3-IFN $\alpha$. Luciferase activity was reduced more effectively after treatment with anti-HER2IgG3-IFN $\alpha$ across a broad range of therapeutic doses (Figure 1B, right panel). Importantly, the difference be- 
A

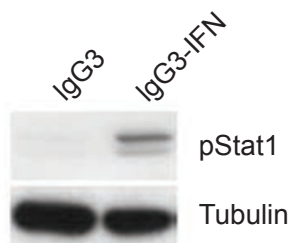

B $\quad:$ IgG3-IFN

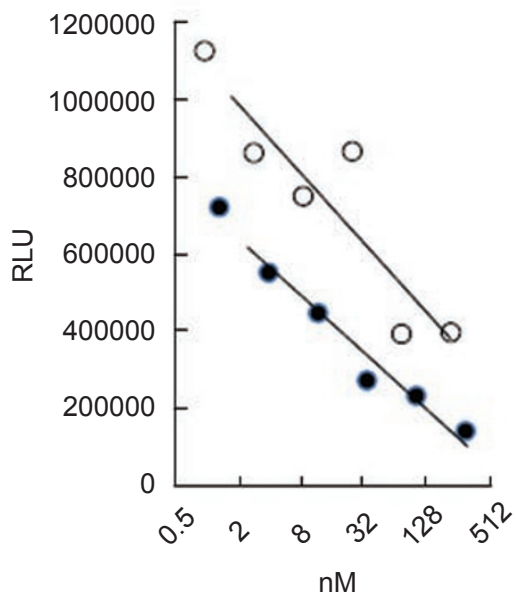

C
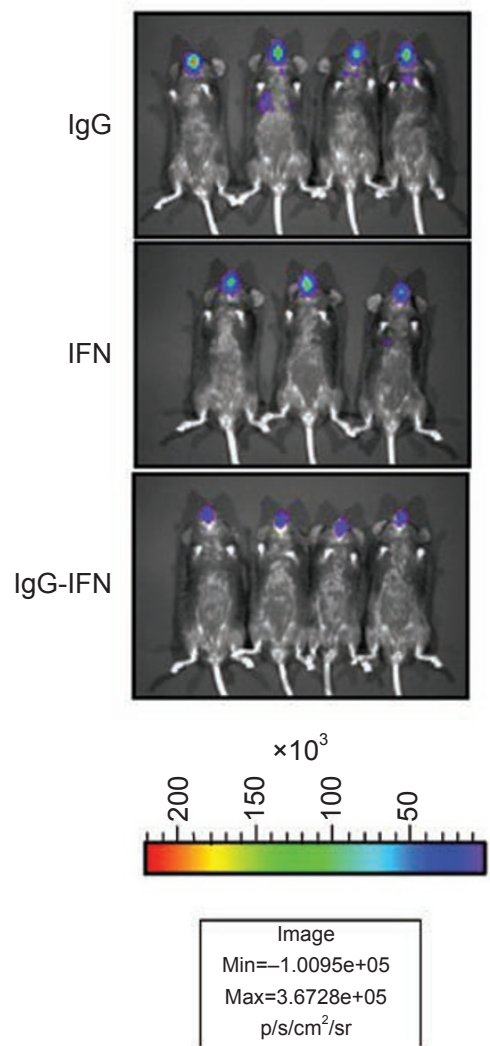

D

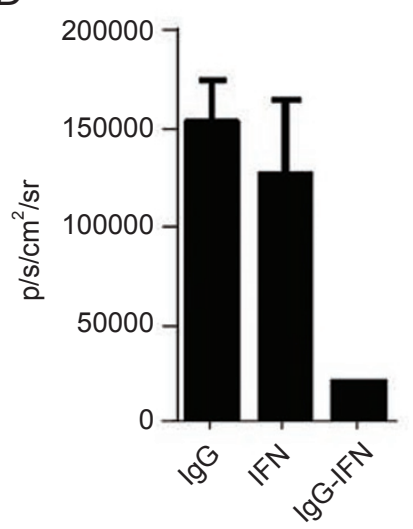

$E$

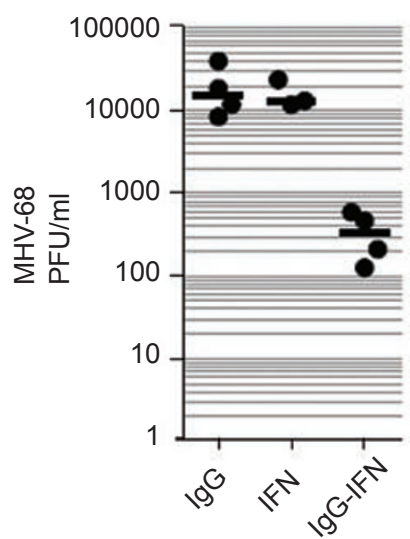

- HER2-IgG3-IFN

○ IgG3-IFN

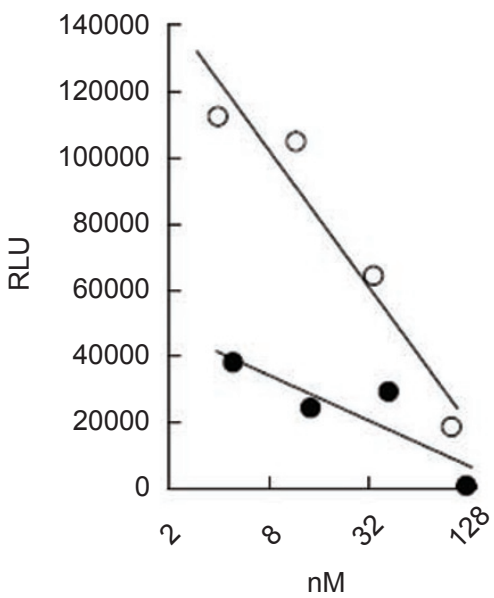

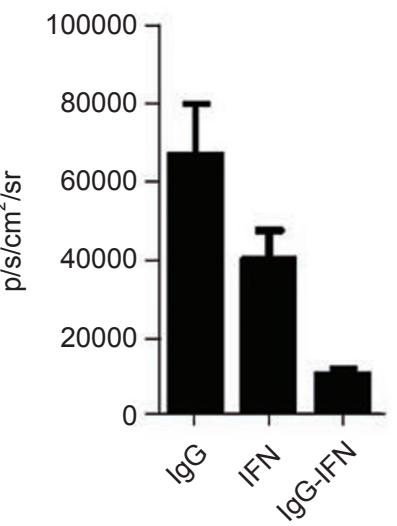

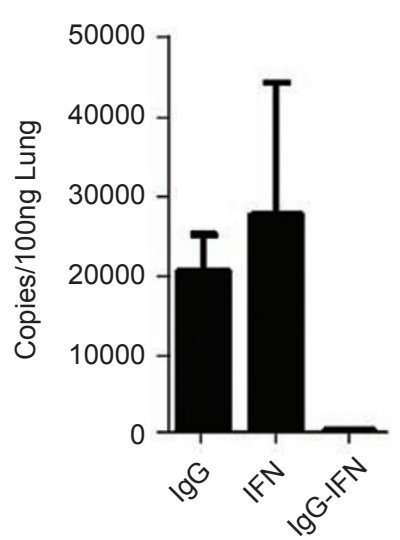

Figure 1 (A) 38C13 cells were stimulated with IgG3-IFN 1 for $30 \mathrm{~min}$, and phosphorylation of STAT1 was assayed by western blot. (B) IgG3-IFN $\alpha$ protects cells from viral infection more effectively than IFN $\alpha$ across a wide range of doses. Anti-HER2IgG3-IFN $\alpha$ offered better antiviral protection than IgG3-IFN $\alpha$ in 38C13-HER2 cells (see text for details). Cells were infected with MHV-68-Luc and treated with the indicated reagents. Cells were harvested $48 \mathrm{~h}$ after infection and assayed for luciferase activity. (C) IgG3-IFN $\alpha$ better inhibited the luciferase expression than IFN $\alpha$ in MHV-68-luc-infected mice. Mice were infected with 5000 PFU of the virus and treated with the indicated reagents i.p. Bioluminescence was measured on day 5 after infection. (D) Bioluminescence readings of IgG3-IFN $\alpha$-treated animals showed a statistically significant reduction in luciferase expression compared with the IFN $\alpha$ group in all measured planes (supine and one lateral plane are graphed). (E) On day 7 after infection, mice were euthanized and lung homogenates were assayed for viral load by plaque assays and q-PCR analysis. MHV-68 viral titers are effectively reduced in the lungs of IgG3-IFN $\alpha$-treated animals compared with those treated with IFN $\alpha$ or IgG alone. 
tween anti-HER2-IgG3-IFN $\alpha$ and IgG3-IFN $\alpha$ was more prominent at low concentrations, suggesting that antiHER2-IgG3-IFN $\alpha$ increases the effectiveness of IFN $\alpha$ by targeting IFN $\alpha$ to HER2/neu-expressing cells. When parental 38C13 cells that did not express HER2/neu were used, both fusion proteins similarly inhibited viral replication (data not shown).

We used an intranasal model of infection with the MHV-68-Luc virus, followed by bioluminescence imaging, to determine the effectiveness of IgG3-IFNa in inhibiting viral replication in vivo. We first administered 5000 PFU of MHV-68-Luc through nasal passages and then treated these mice intraperitoneally with 25000 units of IFN $\alpha, 25000$ units of IgG3-IFN $\alpha(10 \mu \mathrm{g})$, or 10 $\mu \mathrm{g}$ of $\mathrm{IgG} 3$ alone. Mice were imaged on day 5 (Figure 1C). Bioluminescence readings of mice imaged in supine and lateral positions were obtained and are presented in the left and right panels of Figure 1D. Whereas mice treated with IFN $\alpha$ exhibited a slight reduction in bioluminescence readings compared with IgG-treated animals, IgG3-IFN $\alpha$-treated animals exhibited a statistically significant seven-fold reduction in readings obtained in either position $(P<0.0001$ compared with the IgG-treated group). Indeed, mice treated with IgG-IFN had significantly reduced bioluminescence readings compared with the IFN $\alpha$-treated mice $(P<0.05)$.

On day 7 after infection with MHV-68, mice in each of the three groups were euthanized, and viral burden in the lung was measured by plaque assays using lung homogenates. qPCR analysis was also used to determine the copy number of the viral genome in the lungs of infected animals. IgG3-IFN $\alpha$-treated animals exhibited a 100 -fold reduction in viral burden as measured by the plaque assay $(P<0.05$, Student's $t$-test $)$. Surprisingly, treatment with IFN $\alpha$ provided no protection against viral burden as measured by this assay (Figure 1E, left panel). Similarly, viral genome content in the lungs of IgG3-
IFN $\alpha$-treated animals was 600 times lower than that observed in IgG-treated animals, while IFN $\alpha$ treatment seemed insufficient to suppress viral genome production (Figure 1E, right panel). Thus, IgG3-IFN $\alpha$ proved to be a more potent antiviral agent both in vitro and in vivo.

\section{Acknowledgments}

This work was supported by NIH T32 AI00732319 and GM 08042 (AS), and NIH 1R01AI056154 \& 1R01AI069120 (GC), USA.

\section{References}

1 Isaacs A, Lindenmann J. Virus interference. I. The interferon. By A. Isaacs and J. Lindenmann, 1957. J Interferon Res 1987; 7:429-438.

2 Muller U, Steinhoff U, Reis LF, et al. Functional role of type I and type II interferons in antiviral defense. Science 1994; 264:1918-1921.

3 Korenman J, Baker B, Waggoner J, et al. Long-term remission of chronic hepatitis B after alpha-interferon therapy. Ann Intern Med 1991; 114:629-634.

4 Shindo M, Di Bisceglie AM, Cheung L, et al. Decrease in serum hepatitis $\mathrm{C}$ viral RNA during alpha-interferon therapy for chronic hepatitis C. Ann Intern Med 1991; 115:700-704.

5 Kirkwood JM, Strawderman MH, Ernstoff MS, et al. Interferon alfa-2b adjuvant therapy of high-risk resected cutaneous melanoma: the Eastern Cooperative Oncology Group Trial EST 1684. J Clin Oncol 1996; 14:7-17.

6 Davis TA, Maloney DG, Grillo-Lopez AJ, et al. Combination immunotherapy of relapsed or refractory low-grade or follicular non-Hodgkin's lymphoma with rituximab and interferonalpha-2a. Clin Cancer Res 2000; 6:2644-2652.

7 Golomb HM, Jacobs A, Fefer A, et al. Alpha-2 interferon therapy of hairy-cell leukemia: a multicenter study of 64 patients. J Clin Oncol 1986; 4:900-905.

8 Huang T-H, Chintalacharuvu KR, Morrison SL. Targeting IFN-alpha to B cell lymphoma by a tumor-specific antibody elicits potent antitumor activities. $J$ Immunol 2007; 179:68816888 